ARTICLE

\title{
Self-selective formation of ordered 1D and 2D GaBi structures on wurtzite GaAs nanowire surfaces
}

Yi Liu (1) ${ }^{1}$, Johan V. Knutsson", Nathaniel Wilson ${ }^{2}$, Elliot Young ${ }^{2}$, Sebastian Lehmann (1) ${ }^{1}$, Kimberly A. Dick ${ }^{3}$, Chris J. Palmstrøm ${ }^{2,4}$, Anders Mikkelsen ${ }^{1} \&$ Rainer Timm (1) ${ }^{1 凶}$

Scaling down material synthesis to crystalline structures only few atoms in size and precisely positioned in device configurations remains highly challenging, but is crucial for new applications e.g., in quantum computing. We propose to use the sidewall facets of larger III-V semiconductor nanowires (NWs), with controllable axial stacking of different crystal phases, as templates for site-selective growth of ordered few atoms 1D and 2D structures. We demonstrate this concept of self-selective growth by $\mathrm{Bi}$ deposition and incorporation into the surfaces of GaAs NWs to form GaBi structures. Using low temperature scanning tunneling microscopy (STM), we observe the crystal structure dependent self-selective growth process, where ordered 1D GaBi atomic chains and 2D islands are alloyed into surfaces of the wurtzite (Wz) $\{11 \overline{2} 0\}$ crystal facets. The formation and lateral extension of these surface structures are controlled by the crystal structure and surface morphology uniquely found in NWs. This allows versatile high precision design of structures with predicted novel topological nature, by using the ability of NW heterostructure variations over orders of magnitude in dimensions with atomic-scale precision as well as controllably positioning in larger device structures.

\footnotetext{
${ }^{1}$ Department of Physics and NanoLund, Lund University, Lund, Sweden. ${ }^{2}$ Materials Department, University of California-Santa Barbara, Santa Barbara, CA USA. ${ }^{3}$ Centre for Analysis and Synthesis and NanoLund, Lund University, Lund, Sweden. ${ }^{4}$ Department of Electrical and Computer Engineering, University of California-Santa Barbara, Santa Barbara, CA, USA. ${ }^{凶}$ email: rainer.timm@sljus.lu.se
} 
S ite-selected formation of semiconductor nanostructures has so far mainly been realized by two types of approaches: Nanostructures with full control over the spatial position of each atom have been produced by means of atom manipulation ${ }^{1}$, where individual atoms are placed or re-positioned on a clean semiconductor surface using an STM probe tip. In this way, e.g., single atom transistors based on P doping atoms in Si have been demonstrated $^{2,3}$, and quantum dots (QDs) formed of chains of In atoms on an InAs surface have been studied ${ }^{4}$. While these approaches give fascinating insight into quantum processes, they are not suitable for large-scale industrial device processing, and demanding to implement even for scientific purposes. Scalable approaches, on the other hand, are typically based on complex processing recipes using lithography and etching steps ${ }^{5}$. Many impressive examples can be found, including InAs nanoribbons based on multi-step epitaxial lift-off and overgrowth demonstrating high-performance transistor behavior ${ }^{6}$, or self-assembled QDs aligned in chains by substrate strain engineering ${ }^{7}$ or cleavededge overgrowth ${ }^{8}$, aiming for optoelectronic application. In these approaches, however, the lateral positioning is limited by the spatial resolution of the lithographic processes, and the nanostructures formed on top of the substrates are at least several nm high and contain millions of atoms, thus being far from atomicscale precision, which has to be the ultimate goal for quantum devices ${ }^{9}$. 2D small islands and $1 \mathrm{D}$ chains of atoms have been grown on macroscopically homogeneous group IV semiconductor surfaces ${ }^{10-12}$. However, formation of such nanostructures with precise spatial control on III-V or other compound semiconductor heterostructures remains an open challenge.

In an alternative approach, we use side facets of crystal phase engineered III-V semiconductor $\mathrm{NWs}^{13}$ as templates for site-selected overgrowth, after mechanical transfer of the NWs from the growth sample onto a suitable substrate ${ }^{14}$. Such NW heterostructures can be grown with atomically sharp transitions between cubic zincblende $(\mathrm{Zb})$ and hexagonal $\mathrm{Wz}$ segments and tunable length of the corresponding segments, providing the desired spatial precision and control. The wires themselves can be placed intentionally in devices after or even during growth ${ }^{15}$. Interestingly, the various surface facets of both $\mathrm{Zb}$ and $\mathrm{Wz}$ crystal phase ${ }^{13,16-18}$ of III-V semiconductor NWs offer an extra degree of freedom for surface-related growth phenomena. Previous STM studies have successfully resolved the atomic structure of these facets and the $\mathrm{Wz} / \mathrm{Zb}$ interface on $\mathrm{GaAs}^{14,19}$ and other III-V $16,17,20$ NWs, showing that the Zb $\{110\}, W z\{11 \overline{2} 0\}$, and $\mathrm{Wz}\{10 \overline{1} 0\}$ facets are non-polar and usually appear unreconstructed. Step density and surface morphology can also be controlled. However, previous studies have not found any significant ordered incorporation in the atomic lattice ${ }^{18}$.

Among the various possible material combinations of the III-V semiconductor toolbox, alloys with a high bismuth content have recently gained much attention, mainly due to their large spin-orbit splitting and resulting material properties. $\mathrm{GaBi}$ compounds, for example, have been predicted to show band inversion and topological behavior ${ }^{21,22}$. However, III-V alloys with a high Bi content ( $>20 \%$ of the group- $\mathrm{V}$ atoms) could not yet be realized 23,24 , and especially GaBi has been considered as thermodynamically unstable $\mathrm{e}^{25-27}$, while $\mathrm{Bi}$ is also known to act as surfactant in the GaAs/InAs material system ${ }^{28}$. Alternatively, one can consider to deposit $\mathrm{Bi}$ atoms onto the surfaces of binary III-V compounds and thus obtain a high $\mathrm{Bi}$ concentration at the surface. So far, Bi deposition on the non-polar GaAs(110) surface was reported to result in the growth of terraces and islands of pure $\mathrm{Bi}^{29}$, while after $\mathrm{Bi}$ deposition on polar surfaces such as $\mathrm{GaAs}(001)$ or $\{111\}$ mainly Bi-terminated surface reconstructions were observed (see also Supplementary Note 1).
Here, we report the study of $\mathrm{Bi}$ incorporation in the surface of GaAs NWs where we find ordered 1D GaBi chains and 2D GaBi islands, which form self-selectively on the Wz $\{11 \overline{2} 0\}$-type NW facets, in contrast to randomly distributed $\mathrm{Bi}$ atoms in the $\mathrm{Zb}$ $\{110\}$ facets, which are found also in bulk. The present study focuses on the materials aspects of position controlled growth of few atom small nanostructures, but the surface nanostructures observed here are also highly promising for application in quantum information, since they provide local areas of pure $\mathrm{GaBi}$. Still, the main point is how their self-selective formation only on $\mathrm{Wz}$ segments, in combination with the tunability of the structure of the NW template, opens the path towards monolayer thin nanostructures with variable width, atomically sharp borders, and promise for exotic electronic phases of matter.

\section{Results}

Bi atoms incorporation into GaAs NW surface. GaAs NWs (see Fig. 1a and Supplementary Fig. 1) were grown by metal-organic vapor phase epitaxy (MOVPE) via Au particle-assisted growth ${ }^{30}$. The NWs consist of a long Wz segment followed by a Zb segment including smaller $\mathrm{Wz}$ insertions as well, as seen in Fig. 1a. The NWs have been broken off and transferred onto GaAs $\{111\} \mathrm{B}$ substrates, exposing their side facets to the STM tip as sketched in Fig. 1b. Surface morphology has been studied with atomic resolution using STM at $5 \mathrm{~K}$ in ultrahigh vacuum (UHV). Prior to the studies, the native oxide was removed from the NW surfaces by annealing in atomic hydrogen ${ }^{18}$. In order to investigate $\mathrm{Bi}$ incorporation into the GaAs surface, we deposited elemental $\mathrm{Bi}$ from a thermal evaporator onto NW samples annealed to $250^{\circ} \mathrm{C}$. We found that the majority of NWs had the $\{11 \overline{2} 0\}$ - and $\{110\}$ type facets exposed in $\mathrm{Wz}$ and $\mathrm{Zb}$ segments, respectively, while the more seldom $\{10 \overline{1} 0\}$-type facets were observed at a 30 -degree angle inclined to normal. An overview and a zoom-in STM image of a $\{11 \overline{2} 0\} /\{110\}$-type facet with an atomically sharp Wz/Zb interface are shown in Fig. 1c, d, respectively. Terraces with monolayer high surface steps were found on all facets, showing unreconstructed morphology which has the same patterns as expected from oxide-free pure GaAs $\mathrm{NWs}^{14}$, indicating no effect from $\mathrm{Bi}$ incorporation on the general surface morphology (see Supplementary Fig. 2).

$\mathrm{Bi}$ atoms surface incorporation can be found on both $\mathrm{Zb}$ and Wz facets, as shown in Fig. 1c, d. These STM images are obtained at negative sample bias which corresponds to filled state imaging, revealing the group- $\mathrm{V}$ atoms in the top most surface layer ${ }^{31}$. Importantly, a number of bright sites can be seen within the rows of As atoms on the surface after $\mathrm{Bi}$ deposition. Such bright protrusions are consistent with the presence of an adatom or different atomic species incorporated within the atomic lattice ${ }^{32}$, and we associate them with individual $\mathrm{Bi}$ atoms. These $\mathrm{Bi}$ atoms protrude the surrounding GaAs surface by only about $40-50 \mathrm{pm}$, as shown by the line profiles of Fig. 1e for $\{110\}$ - and $\{11 \overline{2} 0\}$-type facets as well as Supplementary Fig. 3 for $\{10 \overline{1} 0\}$-type facets, independent of tunneling voltage and current settings. Thus, we can exclude the influence from the variation of local density of states (LDOS), and conclude the protrusion indeed comes from tomographic changing. Considering the well-studied group- $\mathrm{V}$ exchange reaction, we interpret the brighter protrusions in the As lattice as $\mathrm{Bi}$ atoms that have entered the surface via a Bi-for-As exchange in the top most surface layer, forming local $\mathrm{Ga}-\mathrm{Bi}$ bonds. The brighter contrast of the $\mathrm{Bi}$ atoms compared to the surrounding As atoms originates from the larger atomic radius of $\mathrm{Bi}$. Other similar group-V exchange reactions have been observed before, such as $\mathrm{Bi}$ incorporation in As sites in diluted $\mathrm{GaAsBi}^{33}$ and $\mathrm{Sb}$ in $\mathrm{GaAs}^{18}$. Bi adatoms on top of the surface, in contrast, would result in a much larger height. Bi atoms situated below 

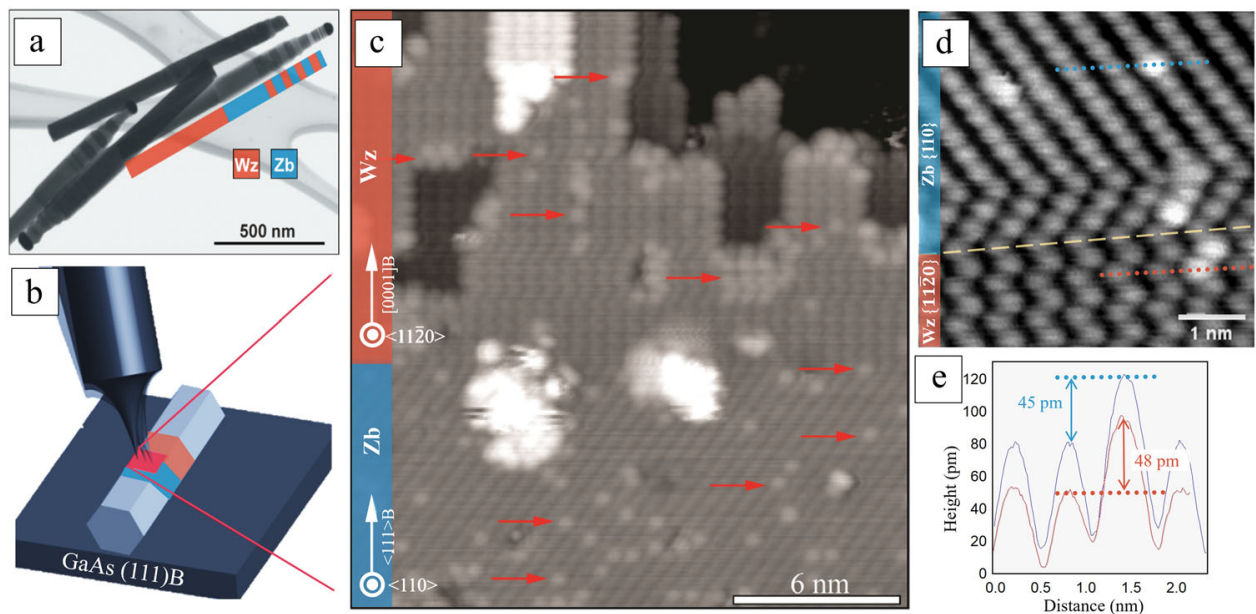

Fig. 1 NW sample preparation and NW surfaces. a Scanning electron microscopy image in transmission mode with crystal phase contrast of GaAs NWs, after transferring onto a suitable sample holder. Brighter and darker contrast corresponds to Zb and Wz, respectively, as confirmed previously by transmission electron microscopy. $\mathbf{b}$ Schematic description of the STM imaging setup showing the STM tip accessing a NW side facet. Orange (blue) color indicates Wz (Zb) segment. The red square demonstrates the area being scanned by STM. c Atomic-scale STM image of a Bi-exposed GaAs NW with a $W z\{11 \overline{2} 0\}$ - (top) and Zb \{110\}- (bottom) type facet, with some Bi atom sites marked by red arrows. Monolayer-high surface steps are found on the $\{11 \overline{2} 0\}$ facet, resulting in terraces of four different heights. Two small bright patches of remaining oxide or adsorbates can be seen close to the Wz-Zb interface. d A zoom-in filled state STM image of the $W z / Z b$ interface, showing the group- $V$ atoms. The yellow dashed line indicates the interface between $W z$ $\{11 \overline{2} 0\}$ - (bottom) and Zb $\{110\}$ - (top) type facet. The brighter dot-like protrusions are Bi incorporation sites, which match well with the As lattice sites. The gray scale range, i.e., the brightest pixel to darkest pixel for (c) is $670 \mathrm{pm}$, and for (d) is $104 \mathrm{pm}$. (e) Line scan along the blue (orange) line in (d) showing the height of the protruded atom in $\mathrm{Zb}(\mathrm{Wz})$ segment. STM images were obtained at $(\mathbf{c}) \mathrm{V}_{\mathrm{T}}=-3.5 \mathrm{~V}, \mathrm{I}_{\mathrm{T}}=100 \mathrm{pA}$ and $(\mathbf{d}) \mathrm{V}_{\mathrm{T}}=-4.4 \mathrm{~V}, \mathrm{I}_{\mathrm{T}}=75 \mathrm{pA}$.

the surface layer would give rise to a much smaller outward relaxation (towards the vacuum), which should still be detectable with STM down to at least 3 bilayers ${ }^{34}$, however, we find no such protrusions. These results suggest Bi-incorporation on $\mathrm{GaAs}$ NWs after growth as a successful approach for achieving local GaBi formation, which has been considered unstable in bulk ${ }^{25-27}$, confined within the top most atomic layer.

Distinct differences in the incorporation of $\mathrm{Bi}$ atoms between the $\mathbf{W z}\{11 \overline{2} 0\}$ - and $\mathrm{Zb}\{110\}$-type facets. On the $\{110\}$-type facets, $\mathrm{Bi}$ atoms are found in a random distribution in the middle of the terraces, as confirmed by autocorrelation analysis (see Supplementary Fig. 4), with some additional Bi agglomeration at step edges (see Fig. 2a). In contrast, very few single Bi sites are observed on the $\{11 \overline{2} 0\}$-type facets, instead a short-range ordering with neighboring sites in all directions is found to be preferable, which results in small $1 \mathrm{D}$ chains or $2 \mathrm{D}$ islands of local $\mathrm{GaBi}$ structures made up with a few tens of atoms (see Fig. 2d); only about $4 \%$ of the Bi atoms were found to sit individually in the $\{11 \overline{2} 0\}$-type surfaces (see Supplementary Fig. 5 and Supplementary Table 1). The atomic lines and zig-zag pattern in Fig. 2a and Fig. $2 \mathrm{~d}$ are due to the specific side facets exposed in NWs, the corresponding crystal structure models can be seen in Figs. 2b and $2 \mathrm{e}$, respectively. It appears favorable for the incorporated $\mathrm{Bi}$ atoms to be positioned next to each other in the nearest neighbor sites on the $\mathrm{Wz}\{11 \overline{2} 0\}$ facet, while Bi prefers to stay at single sites on $\mathrm{Zb}\{110\}$ facets. Statistical results were obtained by analyzing STM images from large areas of the NW facets across five different NWs (see Supplementary Fig. 6 and Supplementary Table 2). It is found that $\mathrm{Bi}$ in average replaces $6 \%, 6 \%$, and $1 \%$ of the As atoms on $\{11 \overline{2} 0\}-,\{110\}-$, and $\{10 \overline{1} 0\}$-type facets, respectively. This is in all cases significantly less than half a monolayer, which was the nominal amount of deposited Bi. This suggests that only a small fraction of the deposited $\mathrm{Bi}$ atoms will overcome the energy barrier for the group- $\mathrm{V}$ exchange reaction and get incorporated into the surface. An example of a single atomic vacancy in the middle of a flat $\{11 \overline{2} 0\}$ terrace, which has triggered formation of a $2 \mathrm{D} \mathrm{GaBi}$ island directly beside it, can be seen in the inset of Fig. 2d. Aggregation of $\mathrm{Bi}$ atoms can also be found surrounding vacancies in the $\{110\}$ terraces, as marked with a red arrow in Fig. 2a. This indicates a much lower exchange energy barrier at the step edges and atomic vacancies as compared to that in a continuous surface, enhancing Bi-for-As exchange. This is due to the generally higher surface potential of As atoms on the higher terrace near the step edge ${ }^{35}$ and around vacancies. Furthermore, $\mathrm{Bi}$ atoms will have more freedom for strain relaxation when incorporating on the step edges.

The mechanism for the different $\mathrm{Bi}$ incorporation on the $\mathrm{Wz}$ and $\mathbf{Z b}$ facets. The behavior can be explained by a model in which $\mathrm{Bi}$, after landing on the surface, diffuses around, upon which it can either substitute with As atoms in the GaAs lattice or evaporate from the surface again. The lower than nominally deposited Bi surface content would support this picture, as not all the $\mathrm{Bi}$ incorporates into the surface. For the $\mathrm{Wz}\{11 \overline{2} 0\}$-type facets a much higher density of $\mathrm{Bi}$ atoms is found on all the step edges facing [0001]A/B directions compared to other step edges (see Fig. 2d). Further STM measurements, performed on $\mathrm{Wz}$ $\{11 \overline{2} 0\}$-type NW facets after Bi deposition resulting in a coverage of less than $10 \%$ (nominally), can be seen in Supplementary Fig. 8. They confirm that the initial sites for Bi incorporation are step edges facing [0001]A/B directions. More specifically, sites on step edges terminated with As atoms (i.e., [0001]B) are more preferable compared to edges terminated with $\mathrm{Ga}$ atoms (i.e., [0001]A). This indicates that the energy barrier is low for Bi-forAs exchange at edges facing [0001]B. 2D GaBi islands or $1 \mathrm{D} \mathrm{GaBi}$ chains are found adjacent to such step edges, where $\mathrm{Bi}$ atoms are occupying neighboring group- $\mathrm{V}$ lattice sites along the [0001]A direction. This cannot be explained by very short range energetics, as the nearest neighbor configuration is identical for the $\mathrm{Wz}\{11 \overline{2} 0\}$ and $\mathrm{Zb}\{110\}$ surface, while ordered $\mathrm{Bi}$ agglomeration is only observed on the $\mathrm{Wz}$ facet. Instead, more long range energetics have to be responsible for the markedly different behavior on the two surfaces. Longer range elastic strain energy 

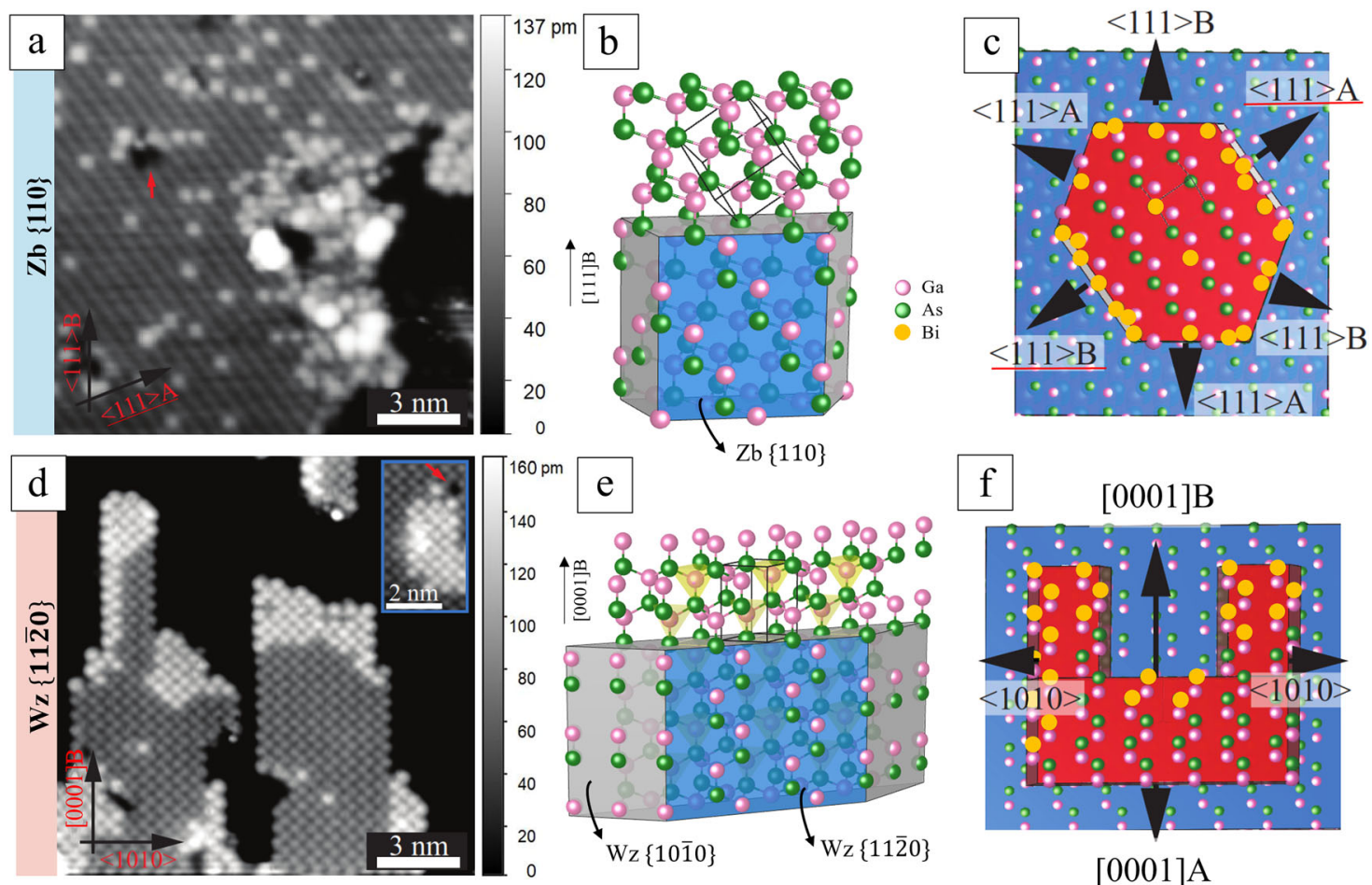

[0001]A

Fig. 2 Bi incorporates into the surface layer of GaAs NWs. a, d shows STM images of the Zb $\{110\}$-type surface and of the $\{11 \overline{2} 0\}$-type surface, respectively, including surface steps along different directions between the top terrace and a one monolayer lower terrace. The lower terraces appear black here due to contrast enhancement, the height scale bars are shown on the right side. Different contrast settings of the same STM image can be found for comparison in Supplementary Fig. 6. The many small bright protrusions correspond to Bi atoms. Red arrows in (a) and (d) point at atomic vacancies. The inset in (d) indicates a 2D GaBi island near a vacancy in the middle of a flat terrace. b, e illustrate the GaAs 3D crystal structure for (b) Zb and (e) Wz phase, overlaid with the NW shape. c, f show 2D models corresponding to the surfaces seen in (a) and (d). The red and blue areas represent the top terrace and the second atomic layer, respectively, of the NW surface, separated by surface steps. An arbitrary shape is chosen for the top terrace, but the possible directions of the surface steps are determined by the crystal structure and correspond to those visible in (a) and (d). Incorporation of Bi atoms (yellow dots) is illustrated, occurring at vacancies and via atomic step edges facing 〈111〉A/B directions on the Zb\{110\}-type surface (c) and step edges facing the [0001] B growth direction on the Wz $\{1120\}$-type surface (f), as motivated by the positions of the Bi atoms observed in (a) and (d). The direction indexes with red underline in $(\mathbf{a})$ and $(\mathbf{c})$ present the projections of $<111>$-type directions in $\{110\}$ plane. In (b), (c), (e), and (f), pink (green/yellow) spheres depict $\mathrm{Ga}(\mathrm{As} / \mathrm{Bi})$ atoms. $V_{T}=-4.4 \mathrm{~V}, I_{T}=100 \mathrm{pA}$ for all STM images.

minimization as a mechanism is not a reasonable explanation either, as the incorporation of several $\mathrm{Bi}$ atoms in close vicinity would lead to a larger overall strain and a broader distribution of Bi near the edges would be preferred, in strong contrast to the almost perfect ordering observed. Here it can further be noted that the lattice difference between $\mathrm{GaBi}$ and $\mathrm{GaAs}$ is larger than for many other more commonly studied systems such as $\mathrm{Si} / \mathrm{Ge}$ and InAs/GaAs. Other possibilities are pure geometric effects ${ }^{36}$ due to limited diffusion on the surface in some directions, but this is also difficult to reconcile with the highly ordered islands observed. Instead, an intermediate situation must be considered in which the zig-zag motif of the top layer of the $\mathrm{Wz}\{11 \overline{2} 0\}$ surface (see Fig. 2f) must lead to a weakening of the Ga-As bonds of the As atoms placed next to the recently incorporated Bi atoms, thus the barrier for incorporation in an adjacent site would be lowered. In contrast, the rows on the $\mathrm{Zb}\{110\}$ surface have a different symmetry (when considering the formation of a cluster of three $\mathrm{Bi}$ atoms or more), which then cannot be energetically favorable. Interestingly, the pronounced effect of next nearest neighbor interaction is fundamental to crystal formation, it is the basic distinguishing factor between $\mathrm{Wz}$ and $\mathrm{Zb}$ crystal structures.

Bi can also exchange As surface atoms in the middle of a $\{11-$ $20\}$ terrace, however, this process has a much lower probability and will only occur for large terraces and depend on temperature, as it is competing with evaporation and edge integration (as seen in Supplementary Fig. 5). The model would then also explain why agglomeration does not start to occur in the presence of a single $\mathrm{Bi}$ atom in the middle of the surface. Morphologically, monolayer high terraces are generally favored elongated along the [0001]A/B directions on the naturally formed $\mathrm{Wz}\{11-20\}$ facets on GaAs and InAs $\mathrm{NWs}^{14,17,18}$. 2D islands result from $\mathrm{Bi}$ incorporation at all group- $\mathrm{V}$ atom positions along a step edge facing [0001]B with subsequent lowering of energetics for $\mathrm{Bi}$ incorporation in adjacent sites. If a $\mathrm{Bi}$ atom is incorporated at a corner site, it appears especially favorable for another $\mathrm{Bi}$ atom to substitute As along a step edge facing a $\langle 10 \overline{1} 0\rangle$ direction, and a $1 \mathrm{D}$ chain is formed along the edge. 1D chains might energetically be favorable over 2D islands due to strain relaxation at the edge. Vacancies in principle trigger Bi incorporation in the same way, as they can generate step edges facing different directions (as seen in the inset of Fig. $2 d$ ). On the $\{110\}$-type facets, Bi-for-As exchange occurs at all step edges, but adjacent group- $\mathrm{V}$ lattice sites are not preferred for subsequent Bi incorporation. Instead adsorption in random sites on the terrace becomes relatively more likely, as well as $\mathrm{Bi}-\mathrm{Bi}$ clustering, where $\mathrm{Bi}$ atoms are incorporated outside group- $\mathrm{V}$ lattice sites. This is in agreement with the lack of large ordered $\mathrm{GaBi}$ structures or GaAsBi alloys with high $\mathrm{Bi}$ content reported previously for the standard $\mathrm{Zb}$ surfaces $^{37}$. While this qualitative model explains the observed results, it also indicates that future detailed calculations of the surface energetics would be highly interesting and could be used to tailor the behavior. 


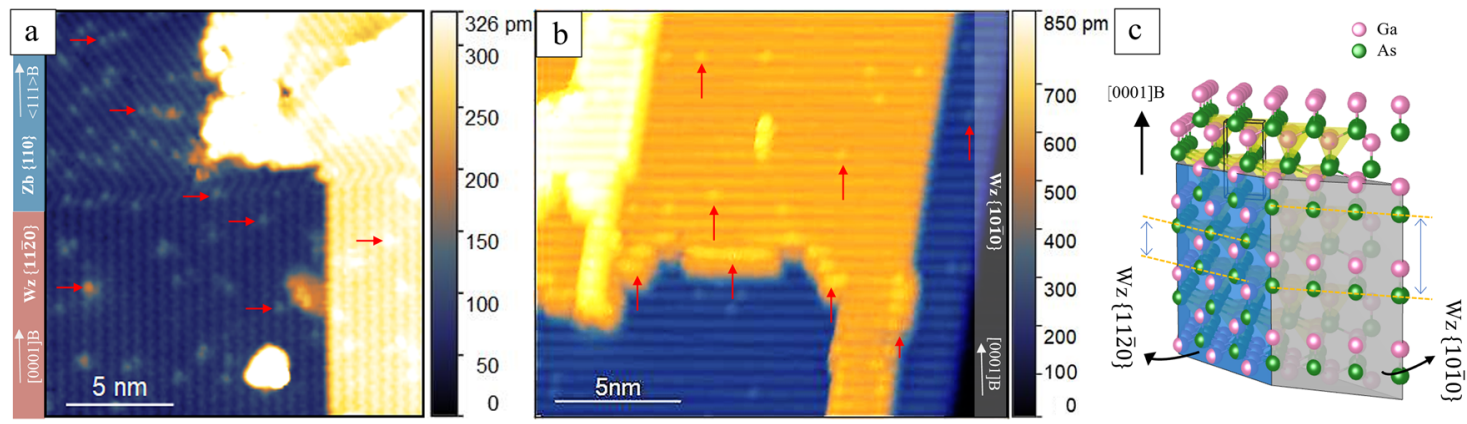

Fig. 3 Atomically resolved STM images of Bi incorporation in GaAs NW surfaces with a lower step density. a shows the interface between a Wz $\{1120\}-$ (bottom) and a Zb \{110\}- (top) type facet. In this image, no step edge facing [0001]A/B directions is present on the Wz $\{11 \overline{2} 0\}$ facet. Almost all Bi atoms are incorporated at individual sites. In contrast, the $\mathrm{Zb}\{110\}$ facet includes more $\mathrm{Bi}$ atoms, in the form of unordered Bi clusters along the step edges or random individual $\mathrm{Bi}$ incorporation sites. $\mathbf{b}$ shows the surface of a Wz $\{10 \overline{1} 0\}$-type facet. At all step edges facing [0001]-type directions, most of the As atoms are substituted by $\mathrm{Bi}$, while very few $\mathrm{Bi}$ incorporation sites are found on the other step edges or within the terraces. The slightly tilted appearance of the step edges is explained in Supplementary Note 2. Some Bi atoms are indicated in (a) and (b) by red arrows. The height scale bars are shown on the right side in both (a) and (b). c sketches the $30^{\circ}$ edge between a Wz $\{10 \overline{1} 0\}$ - and a $\{11 \overline{2} 0\}$ - type facet, pink (green) spheres depict Ga (As) atoms. Every layer of $\mathrm{Ga}$ and $\mathrm{As}$ (or $\mathrm{Bi}$ ) atoms along the [0001]B growth direction can be seen on the $\{11 \overline{2} 0\}$ surface, resulting in the zig-zag chain pattern visible at high resolution as e.g., in Fig. $2 \mathrm{~d}$ due to the $\mathrm{AB}$-stacking sequence of the atomic layers. However, only every second layer of $\mathrm{Ga}$ and $\mathrm{As}$ (or $\mathrm{Bi}$ ) atoms can be seen in the top most plane of the $\{10 \overline{10}\}$ surface. STM images were obtained at (a) $\mathrm{V}_{\mathrm{T}}=-4.4 \mathrm{~V}, \mathrm{I}_{\mathrm{T}}=50 \mathrm{pA}$ and $(\mathbf{b}) \mathrm{V}_{\mathrm{T}}=-4.5 \mathrm{~V}, \mathrm{I}_{\mathrm{T}}=90 \mathrm{pA}$.

Bi incorporation on GaAs NW surface with lower step density. To further investigate the influence of surface orientation and step density on the Bi incorporation, we studied another type of GaAs NWs with larger surface terraces and a lower step density. An STM image of an interface of $\{11 \overline{2} 0\} /\{110\}$-type facets on such a GaAs NW is shown in Fig. 3a, which presents a similar distribution of $\mathrm{Bi}$ atoms on the $\mathrm{Zb}\{110\}$ facet as before (Figs. 1c and 2a), but a significantly different configuration for the Wz $\{11 \overline{2} 0\}$ facet (compared with Figs. $1 \mathrm{C}$ and 2C): Here, incorporated Bi atoms are only found at individual sites, which appear to be randomly distributed. The only step edges, which are facing the $<10 \overline{1} 0>$-type directions, show the same low density of Bi sites as the flat terraces, without any short-range ordering of $\mathrm{Bi}$ atoms. Furthermore, here in average $7 \%$ of the surface As atoms were replaced by $\mathrm{Bi}$ on the $\{110\}$-type facets, but only $3 \%$ on the $\{11 \overline{2} 0\}$-type facets, while the ratio was the same for both types of facets for the NWs with higher step densities (see Supplementary Fig. 7 and Supplementary Table 3). These results confirm that the step edges facing the [0001]A/B directions on $\mathrm{Wz}\{11 \overline{2} 0\}$ facet, which are missing here due to the larger terrace size, play a critical role on triggering $\mathrm{Bi}$ incorporation and forming ordered $\mathrm{GaBi}$ structures.

Yet another behavior of $\mathrm{Bi}$ incorporation is observed on $\mathrm{Wz}$ $\{10 \overline{1} 0\}$-type facets, as shown in Fig. 3b. Although Bi atoms successfully incorporate into the first sites on the step edges facing [0001]A/B directions, no ordered $\mathrm{GaBi}$ structures extending further into the terraces can be found. We attribute this different $\mathrm{Bi}$ incorporation to the different surface structure of $\mathrm{Wz}\{11 \overline{2} 0\}$ and $\mathrm{Wz}\{10 \overline{1} 0\}$ facets, as shown in the model of Fig. 3c. On the $\mathrm{Wz}\{10 \overline{1} 0\}$ surface, the horizontal As (or $\mathrm{Ga}$ ) atomic chains are separated along the [0001]A/B directions by $0.55 \mathrm{~nm}$, which is twice the distance between neighboring As (or $\mathrm{Ga}$ ) atoms along the [0001]A/B direction in the zig-zag motif on the $\mathrm{Wz}\{11 \overline{2} 0\}$ surface. The much larger gap results in a smaller nearest neighbor effect of $\mathrm{Bi}$ atoms along [0001]A/B directions on the $\{1010\}$ facet. This is consistent with the $\mathrm{Bi}$ incorporation mechanism discussed above.

Finally, we want to further explore the conditions under which $\mathrm{Bi}$ incorporation into the sample surface can occur at all. We found that if the sample is at too low temperature (e.g., room temperature) during $\mathrm{Bi}$ deposition, only metallic Bi clusters will be formed on the surface. On the contrary, if the sample temperature is too high (e.g., $400^{\circ} \mathrm{C}$ ), $\mathrm{Bi}$ atoms tend to evaporate directly when landing on the GaAs surface, thus resulting in a very low Bi deposition and incorporation rate. Synchrotron based nano-focused X-ray photoelectron spectroscopy (XPS) has been performed to study the $\mathrm{Bi}$ incorporation process as well, as shown in Supplementary Fig. 9. The presence of $\mathrm{Bi}$ atoms in the NW surface after Bi deposition was confirmed from Bi $4 \mathrm{f}$ core level spectra. For these experiments, GaAs NWs have been transferred onto $\mathrm{Si}$ substrates prior to Bi deposition. Interestingly, 2D maps obtained by scanning photoelectron microscopy (SPEM), shown in Supplementary Fig. 9, demonstrate a much higher Bi signal at the GaAs NW as compared with the surrounding Si substrate. This can be explained by the $\mathrm{Bi}$ incorporation process on NWs, such that the incorporated $\mathrm{Bi}$ atoms remain on the NW, while the $\mathrm{Bi}$ atoms landing on the Si substrate tend to get directly desorbed again from the surface.

We conclude that the crystal-phase selective $\mathrm{Bi}$ incorporation on GaAs NWs is a result of the interplay between the atomic morphology and the surface energy of the different NW facets. In our model, the self-selected diffusion paths and incorporation sites for $\mathrm{Bi}$ atoms are determined by the surface energy and the next nearest neighbor geometry. By carefully tailoring $\mathrm{Wz} / \mathrm{Zb}$ heterostructure NWs, unique 2D and $1 \mathrm{D}$ nanostructures of pure $\mathrm{GaBi}$ which are fully confined to one atomic layer can be achieved, growing self-selectively on the NW $\{11 \overline{2} 0\}$ facets. A high density of step edges facing [0001]A/B directions is found essential to trigger the controlled $\mathrm{Bi}$ incorporation. The crystal phase and surface step engineering of NW facets are inspiring for realization of well-positioned nanostructures and QDs down to atomic scale. What's more, the study indicates a promising approach to realize stable and ordered GaBi surface alloys as well as $3 \mathrm{D} \mathrm{GaBi}$ nanostructures with atomic-scale precision by using tailored NW-based templates and radial overgrowth, which is an important step towards Bi-based III-V nanostructures with novel topologic and electronic properties.

\section{Methods}

NW growth. GaAs NWs were prepared by metal-organic vapor phase epitaxy (MOVPE) using a $3 \times 2$ " Aixtron close-coupled showerhead reactor (CCS) at a total reactor flow of $8 \mathrm{slm}$, a total reactor pressure of $100 \mathrm{mbar}$, and hydrogen as carrier gas. Au aerosol particles with a total areal density of $1 \mu \mathrm{m}^{-2}$ and nominal diameters of 30,50 , and $70 \mathrm{~nm}$ were deposited onto GaAs( $\overline{1} \overline{1} \overline{1})$ substrates by aerosol technique ${ }^{30}$ in order to enable the particle-assisted NW growth mode. After 
annealing the substrates at a set temperature of $630^{\circ} \mathrm{C}$ for $10 \mathrm{~min}$ in an $\mathrm{AsH}_{3} / \mathrm{H}_{2}$ atmosphere, a step necessary to remove surface oxides and allow for a proper surface conditioning, the temperature was set to $550^{\circ} \mathrm{C}$ for NW growth. The precursor materials trimethylgallium $(\mathrm{TMGa})$ and arsine $\left(\mathrm{AsH}_{3}\right)$ were set to molar fractions of $\chi_{T M G a}=1.9 \times 10^{-5}$ as well as $\chi_{A s H 3}=4.5 \times 10^{-5}$ and $4.4 \times 10^{-3}$ for Wz and $\mathrm{Zb}$ conditions, respectively, and, after a temperature stabilization step, introduced into the reactor chamber in order to initialize NW growth. Heterostructured NWs consisting of alternating segments of $\mathrm{Wz}$ and $\mathrm{Zb}$, specifically designed for the experiments, were engineered by only switching the group- $\mathrm{V}$ precursor flow. For detailed information about the growth of sharp crystal structure interfaces in III-V NW systems, the reader is kindly referred to ref. ${ }^{38}$ and references therein. In the presented work the focus was on two types of heterostructured GaAs NWs. Type I had Wz facets of a length of around $43 \mathrm{~nm}$ with many small terraces on the NW facets of typically $2-8 \mathrm{~nm}$ resulting in a high density of atomic surface steps. Type II sample instead had WZ facets of a length of around $35 \mathrm{~nm}$ with terraces typically larger than $8 \mathrm{~nm}$, corresponding to significantly fewer atomic surface steps. Here the terrace size refers to the length of a surface terrace along the direction perpendicular to the NW growth direction. Scanning electron microscopy (SEM) for morphological and structural characterization was carried out in standard and transmission imaging mode in dedicated microscopes (Zeiss Leo Gemini 1560 and Hitachi SU 8010).

Scanning tunneling microscopy. For STM imaging, the NWs were broken off their growth substrates, mechanically transferred onto n-type GaAs ( $(\overline{1} \overline{1})$ substrates ${ }^{14}$, and loaded into the STM chamber with a base pressure of less than $1 \times 10^{-10} \mathrm{mbar}$. After this, the samples were annealed to $500^{\circ} \mathrm{C}$ for $1-3 \mathrm{~h}$ in an atomic hydrogen beam, provided by a thermal cracker (MBE Komponenten) operating at a hydrogen chamber pressure of $2 \times 10^{-6} \mathrm{mbar}$, in order to remove the native oxide which forms on the NW surfaces upon transport in air. This procedure has been proven before to be a suitable way to clean III-V NWs ${ }^{14,16,18}$. The cleaned NW surfaces were studied using an Omicron low-temperature (LT) STM, operated in constant current mode at $5 \mathrm{~K}$. Chosen values of sample bias, $V_{T}$, and tunneling current, $I_{T}$, are indicated in the figure captions. Tungsten tips were employed that had been electrochemically etched and afterward cleaned in UHV by electron bombardment. Significant tip-induced band bending effects were observed; due to dopant freezeout, relatively high sample bias was required upon STM imaging. The Bi deposition was performed on the oxide-free sample, making use of a multi-chamber UHV cluster tool without breaking vacuum, using an effusion cell with a PBN crucible at a sample temperature of $250^{\circ} \mathrm{C}$. The temperature of the Bi cell was set to $420^{\circ} \mathrm{C}$ $\left(450^{\circ} \mathrm{C}\right.$ for the sample with the larger surface terraces), resulting in an atomic Biflux of $4.0(7.8) \times 10^{13}$ atoms $\mathrm{cm}^{-2} \mathrm{~min}^{-1}$. The deposition time was $363 \mathrm{~s}(187 \mathrm{~s})$, corresponding to a deposited $\mathrm{Bi}$ amount of $0.5 \mathrm{ML}$ (for both samples).

\section{Data availability}

The STM and XPS raw data generated in this study are available from the corresponding author upon reasonable request.

\section{Code availability}

No custom code was developed for the analysis of the data of this study.

Received: 1 February 2021; Accepted: 20 September 2021; Published online: 13 October 2021

\section{References}

1. Ternes, M., Lutz, C. P., Hirjibehedin, C. F., Giessibl, F. J. \& Heinrich, A. J. The force needed to move an atom on a surface. Science 319, 1066, https://doi.org/ $10.1126 /$ science.1150288 (2008).

2. Fuechsle, M. et al. A single-atom transistor. Nat. Nanotechnol. 7, 242-246, https://doi.org/10.1038/nnano.2012.21 (2012).

3. He, Y. et al. A two-qubit gate between phosphorus donor electrons in silicon. Nature 571, 371-375, https://doi.org/10.1038/s41586-019-1381-2 (2019).

4. Fölsch, S., Martínez-Blanco, J., Yang, J., Kanisawa, K. \& Erwin, S. C. Quantum dots with single-atom precision. Nat. Nanotechnol. 9, 505-508, https://doi.org/ 10.1038/nnano.2014.129 (2014)

5. del Alamo, J. A. Nanometre-scale electronics with III-V compound semiconductors. Nature 479, 317-323, https://doi.org/10.1038/nature10677 (2011).

6. Ko, H. et al. Ultrathin compound semiconductor on insulator layers for highperformance nanoscale transistors. Nature 468, 286-289, https://doi.org/ 10.1038/nature09541 (2010).

7. Mano, T. et al. Complex quantum dot arrays formed by combination of selforganized anisotropic strain engineering and step engineering on shallow patterned substrates. J. Appl. Phys. 97, 014304, https://doi.org/10.1063/ 1.1823578 (2005).
8. Bauer, J. et al. Long-range ordered self-assembled InAs quantum dots epitaxially grown on (110) GaAs. Appl. Phys. Lett. 85, 4750-4752, https:// doi.org/10.1063/1.1819987 (2004).

9. Palacio-Morales, A. et al. Atomic-scale interface engineering of Majorana edge modes in a 2D magnet-superconductor hybrid system. Sci. Adv. 5, eaav6600 (2019).

10. Dähne, M. et al. in Advances in Solid State Physics 227-238 (Springer, 2001).

11. Crain, J. N. \& Himpsel, F. J. Low-dimensional electronic states at silicon surfaces. Appl. Phys. A 82, 431-438, https://doi.org/10.1007/s00339-005-3365-3 (2006).

12. Tegenkamp, C. et al. Coupled $\mathrm{Pb}$ chains on $\mathrm{Si}(557)$ : origin of one-dimensional conductance. Phys. Rev. Lett. 100, 076802, https://doi.org/10.1103/PhysRevLett. 100.076802 (2008)

13. Jacobsson, D. et al. Interface dynamics and crystal phase switching in GaAs nanowires. Nature 531, 317-322, https://doi.org/10.1038/nature17148 (2016).

14. Hjort, M. et al. Direct imaging of atomic scale structure and electronic properties of GaAs wurtzite and zinc blende nanowire surfaces. Nano Lett. 13, 4492-4498, https://doi.org/10.1021/nl402424x (2013).

15. Barker, D. et al. Individually addressable double quantum dots formed with nanowire polytypes and identified by epitaxial markers. Appl. Phys. Lett. 114, 183502 (2019)

16. Knutsson, J. et al. Atomic scale surface structure and morphology of InAs nanowire crystal superlattices: the effect of epitaxial overgrowth. ACS Appl. Mater. interfaces 7, 5748-5755 (2015).

17. Hjort, M. et al. Electronic and structural differences between wurtzite and zinc blende InAs nanowire surfaces: experiment and theory. ACS Nano $\mathbf{8}$, 12346-12355, https://doi.org/10.1021/nn504795v (2014).

18. Hjort, M. et al. Crystal structure induced preferential surface alloying of $\mathrm{Sb}$ on Wurtzite/zinc blende GaAs nanowires. Nano Lett. 17, 3634-3640, https:// doi.org/10.1021/acs.nanolett.7b00806 (2017).

19. Capiod, P. et al. Band offsets at zincblende-wurtzite GaAs nanowire sidewall surfaces. Appl. Phys. Lett. 103, 122104 (2013).

20. $\mathrm{Xu}, \mathrm{T}$. et al. Faceting, composition and crystal phase evolution in III-V antimonide nanowire heterostructures revealed by combining microscopy techniques. Nanotechnology 23, 095702 (2012).

21. Huang, H., Liu, J. \& Duan, W. Nontrivial Z2 topology in bismuth-based III-V compounds. Phys. Rev. B 90, 195105, https://doi.org/10.1103/ physrevb.90.195105 (2014).

22. Chuang, F.-C. et al. Prediction of large-gap two-dimensional topological insulators consisting of bilayers of group III elements with Bi. Nano Lett. 14, 2505-2508, https://doi.org/10.1021/nl500206u (2014).

23. Wang, S. \& Lu, P. Bismuth-containing Alloys and Nanostructures. Vol. 285 (Springer, 2019)

24. Tait, C. R., Yan, L. \& Millunchick, J. M. Droplet induced compositional inhomogeneities in GaAsBi. Appl. Phys. Lett. 111, 042105, https://doi.org/ 10.1063/1.4996537 (2017).

25. Punkkinen, M. P. J. et al. Thermodynamics of the pseudobinary GaAs1-xBix $(0 \leq x \leq 1)$ alloys studied by different exchange-correlation functionals, special quasi-random structures and Monte Carlo simulations. Computational Condens. Matter 5, 7-13, https://doi.org/10.1016/j.cocom.2015.09.002 (2015).

26. Ferhat, M. \& Zaoui, A. Structural and electronic properties of III-V bismuth compounds. Phys. Rev. B 73, 115107 (2006).

27. Francoeur, S. et al. Band gap of GaAs1-xBix, $0<x<3.6 \%$. Appl Phys. Lett. 82, 3874 (2003)

28. Lewis, R. B. et al. Self-assembly of InAs nanostructures on the sidewalls of GaAs nanowires directed by a Bi surfactant. Nano Lett. 17, 4255-4260, https:// doi.org/10.1021/acs.nanolett.7b01185 (2017).

29. McLean, A., Feenstra, R., Taleb-Ibrahimi, A. \& Ludeke, R. Electronic and structural properties of a discommensurate monolayer system: GaAs (110)(1×1) Bi. Phys. Rev. B 39, 12925 (1989).

30. Magnusson, M. H., Deppert, K., Malm, J.-O., Bovin, J.-O. \& Samuelson, L. Size-selected gold nanoparticles by aerosol technology. Nanostruct. Mater. 12, 45-48, https://doi.org/10.1016/s0965-9773(99)00063-x (1999).

31. Feenstra, R. M., Stroscio, J. A., Tersoff, J. \& Fein, A. P. Atom-selective imaging of the GaAs(110) surface. Phys. Rev. Lett. 58, 1192-1195 (1987).

32. Ebert, H. P. Imaging defects and dopants. Mater. Today 6, 36-43 (2003).

33. Honolka, J. et al. Electronic properties of GaAsBi (001) alloys at low Bi content. Phys. Rev. Mater. 3, 044601 (2019).

34. Krammel, C. M. et al. Incorporation of Bi atoms in InP studied at the atomic scale by cross-sectional scanning tunneling microscopy. Phys. Rev. Mater. 1, 034606 (2017).

35. Salmi, M. A., Alatalo, M., Ala-Nissila, T. \& Nieminen, R. M. Energetics and diffusion paths of gallium and arsenic adatoms on flat and stepped $\mathrm{GaAs}(001)$ surfaces. Surf. Sci. 425, 31-47, https://doi.org/10.1016/s0039-6028(99)00180-6 (1999).

36. Denker, U., Stoffel, M. \& Schmidt, O. G. Probing the lateral composition profile of self-assembled islands. Phys. Rev. Lett. 90, 196102, https://doi.org/ 10.1103/PhysRevLett.90.196102 (2003). 
37. Duzik, A. et al. Surface structure of bismuth terminated GaAs surfaces grown with molecular beam epitaxy. Surf. Sci. 606, 1203-1207 (2012).

38. Lehmann, S., Wallentin, J., Jacobsson, D., Deppert, K. \& Dick, K. A. A general approach for sharp crystal phase switching in InAs, GaAs, InP, and GaP nanowires using only group V flow. Nano Lett. 13, 4099-4105, https://doi.org/ $10.1021 / \mathrm{nl} 401554 \mathrm{w}(2013)$.

\section{Acknowledgements}

This work was performed within the NanoLund Center for Nanoscience at Lund University and was further supported by the Swedish Research Council (VR), grant nos. 2014-04580 and 2017-04108, by the Knut and Alice Wallenberg Foundation (KAW), Grant no. 2017.0061, and by the project CALIPSOplus from the EU Framework Programme HORIZON 2020, Grant Agreement 730872. The STM studies at UCSB were supported by National Science Foundation (NSF) Grant No. DMR-1507875. The authors are grateful to Matteo Amati, Rahul Parmar, and Luca Gregoratti from the Elettra Synchrotron for experimental support.

\section{Author contributions}

Y.L., J.V.K., A.M., and R.T. conceived the idea and developed the layout of the experiment. S.L. grew the NW samples, Y.L., J.V.K., N.W., and E.Y. performed the Bi deposition process and operated the STM system. Y.L. and J.V.K. analyzed the data., R.T., A.M., and C.J.P joined the discussions. Y.L. and J.V.K. prepared the manuscript in collaboration with R.T. All authors discussed the results and commented on the manuscript.

\section{Funding}

Open access funding provided by Lund University.

\section{Competing interests}

The authors declare no competing interests.

\section{Additional information}

Supplementary information The online version contains supplementary material available at https://doi.org/10.1038/s41467-021-26148-4.

Correspondence and requests for materials should be addressed to Rainer Timm.

Peer review information Nature Communications thanks Gilles Patriarche and the other, anonymous, reviewer for their contribution to the peer review of this work. Peer reviewer reports are available.

Reprints and permission information is available at http://www.nature.com/reprints

Publisher's note Springer Nature remains neutral with regard to jurisdictional claims in published maps and institutional affiliations.

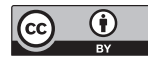

Open Access This article is licensed under a Creative Commons Attribution 4.0 International License, which permits use, sharing, adaptation, distribution and reproduction in any medium or format, as long as you give appropriate credit to the original author(s) and the source, provide a link to the Creative Commons license, and indicate if changes were made. The images or other third party material in this article are included in the article's Creative Commons license, unless indicated otherwise in a credit line to the material. If material is not included in the article's Creative Commons license and your intended use is not permitted by statutory regulation or exceeds the permitted use, you will need to obtain permission directly from the copyright holder. To view a copy of this license, visit http://creativecommons.org/ licenses/by/4.0/.

(C) The Author(s) 2021 